\title{
Article \\ Generalized $k$-Fractional Integral Operators Associated with Pólya-Szegö and Chebyshev Types Inequalities
}

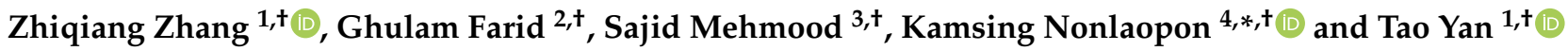 \\ 1 School of Computer Science, Chengdu University, Chengdu 610000, China; zqzhang@cdu.edu.cn (Z.Z.); \\ yantao@cdu.edu.cn (T.Y.) \\ 2 Department of Mathematics, COMSATS University Islamabad, Attock Campus, Attock 43600, Pakistan; \\ faridphdsms@hotmail.com or ghlmfarid@cuiatk.edu.pk \\ 3 Govt Boys Primary School Sherani, Hazro Attock 43440, Pakistan; smjg227@gmail.com \\ 4 Department of Mathematics, Faculty of Science, Khon Kaen University, Khon Kaen 40002, Thailand \\ * Correspondence: nkamsi@kku.ac.th \\ + These authors contributed equally to this work.
}

check for

updates

Citation: Zhang, Z.; Farid, G.;

Mehmood, S.; Nonlaopon, K.; Yan, T.

Generalized $k$-Fractional Integral

Operators Associated with

Pólya-Szegö and Chebyshev Types

Inequalities. Fractal Fract. 2022, 6, 90.

https://doi.org/10.3390/fractalfract 6020090

Academic Editor: Dumitru Baleanu

Received: 15 December 2021

Accepted: 4 February 2022

Published: 6 February 2022

Publisher's Note: MDPI stays neutral with regard to jurisdictional claims in published maps and institutional affiliations.

Copyright: (C) 2022 by the authors Licensee MDPI, Basel, Switzerland. This article is an open access article distributed under the terms and conditions of the Creative Commons Attribution (CC BY) license (https:// creativecommons.org/licenses/by/ $4.0 /)$.

\begin{abstract}
Inequalities related to derivatives and integrals are generalized and extended via fractional order integral and derivative operators. The present paper aims to define an operator containing Mittag-Leffler function in its kernel that leads to deduce many already existing well-known operators. By using this generalized operator, some well-known inequalities are studied. The results of this paper reproduce Chebyshev and Pólya-Szegö type inequalities for Riemann-Liouville and many other fractional integral operators.
\end{abstract}

Keywords: Chebyshev inequality; Pólya-Szegö inequality; fractional integral operators; Mittag-Leffler function

\section{Introduction}

Integral and derivative operators of fractional order are simple and important tools to generalize the classical theories and well-known problems related to integer order derivatives and integrals. Many modern subjects in different fields of mathematics, engineering, and sciences have been introduced due to the applications of fractional derivatives and integrals. These days, fractional integral/derivative operators are very frequently considered by the researchers working on mathematical inequalities to extend the classical literature. One can see the well-known inequalities related to integer order derivatives and integrals have been extended to fractional order derivatives and integrals. These include the inequalities of Chebyshev [1], Hadamard [2], Jensen [3], Pólya-Szegö [4], Petrovic [5], Grüss [6], Ostrowski [7], and many others. Here, we are interested to refer the versions of all these inequalities for Riemann-Liouville fractional integrals studied in [8-13].

It is interesting to compare the integral mean of product of two functions to the product of integral means of these functions. The Chebyshev inequality provides the comparison of integral mean of product of two positive functions of same monotonicity to the product of their integral means. After Chebyshev's inequality, people started to analyze the error bounds of this inequality. For instance, the well-known Grüss inequality gives the error bounds of difference of terms of the Chebyshev inequality (which is well-known as Chebyshev-functional). The well-known Pólya-Szegö inequality gives the estimation of quotient in terms of the Chebyshev inequality for bounded functions. These inequalities have been studied for Riemann-Liouville and other fractional integral operators in [10,14-20]. 
Next, we give the results, which are necessary to produce the results of this paper. First, we give Chebyshev functional and then the Chebyshev inequality [1] as follows:

$$
T(f, g)=\frac{1}{b-a} \int_{a}^{b} f(\tau) g(\tau) d \tau-\left(\frac{1}{b-a} \int_{a}^{b} f(\tau) d \tau\right)\left(\frac{1}{b-a} \int_{a}^{b} g(\tau) d \tau\right),
$$

where $f$ and $g$ are two positive and integrable functions over the interval $[a, b]$. If $f$ and $g$ are synchronous on $[a, b]$, then Chebyshev inequality $T(f, g) \geq 0$ is obtained.

The functional (1) has attracted the attention of many researchers due to its application in mathematical analysis. One of the famous inequalities related to functional (1) is the Grüss inequality [6], stated as follows:

$$
|T(f, g)| \leq \frac{(U-u)(V-v)}{4}
$$

where the positive and integrable functions $f$ and $g$ satisfy

$$
u \leq f(\tau) \leq U, \quad v \leq g(\tau) \leq V,
$$

for all $\tau \in[a, b]$ and constants $u, U, v, V \in \mathbb{R}$.

Another famous inequality which will be useful to obtain our main results is the Pólya-Szegö inequality [4], stated as follows:

$$
\frac{\int_{a}^{b} f^{2}(\tau) d \tau \int_{a}^{b} g^{2}(\tau) d \tau}{\left(\int_{a}^{b} f(\tau) g(\tau) d \tau\right)^{2}} \leq \frac{1}{4}\left(\sqrt{\frac{u v}{u V}}+\sqrt{\frac{U V}{u v}}\right)^{2} .
$$

By using the Pólya-Szegö inequality, Dragomir and Diamond [21] proved the following Grüss type inequality:

$$
|T(f, g)| \leq \frac{(U-u)(V-v)}{4(b-a)^{2} \sqrt{u U v V}} \int_{a}^{b} f(\tau) d \tau \int_{a}^{b} g(\tau) d \tau,
$$

where positive and integrable functions $f$ and $g$ satisfy

$$
0<u \leq f(\tau) \leq U<\infty, \quad 0<v \leq g(\tau) \leq V<\infty,
$$

for all $\tau \in[a, b]$ and constants $u, U, v, V \in \mathbb{R}$.

Inspired by the above-defined inequalities, our aim in this paper is to get some fractional versions of these inequalities. The main objective is to give some new PólyaSzegö and Chebyshev inequalities for generalized $k$-fractional integral operator containing Mittag-Leffler function in its kernel. In the upcoming section, we define a new $k$-fractional integral operator containing Mittag-Leffer function. In Section 3, we will utilize this $k$-fractional integral operator to obtain the Pólya-Szegö and Chebyshev inequalities. Moreover, the presented results are the generalizations of the results which are already published in $[10,14,19]$.

\section{Fractional Integral Operators}

Fractional integral operators are very useful in the advancement of mathematical inequalities. A large number of fractional integral inequalities due to different types of fractional integral operators have been established (see [10,14,19,22-29] and references therein). Applications of fractional integral operators in differential equations and other fields can be found in [25,30-35]. The first formulation of fractional integral operator is the Riemann-Liouville fractional integral operator, defined as follows: 
Definition 1. Let $f \in L_{1}[a, b]$. Then Riemann-Liouville fractional integrals of order $\sigma \in \mathbb{C}$, $\Re(\sigma)>0$ are defined by:

$$
\begin{aligned}
& \left(\xi_{a^{+}}^{\sigma} f\right)(x)=\frac{1}{\Gamma(\sigma)} \int_{a}^{x}(x-\tau)^{\sigma-1} f(\tau) d \tau, \quad x>a \\
& \left(\xi_{b^{-}}^{\sigma} f\right)(x)=\frac{1}{\Gamma(\sigma)} \int_{x}^{b}(\tau-x)^{\sigma-1} f(\tau) d \tau, \quad x<b,
\end{aligned}
$$

where $\Gamma($.$) is the gamma function defined as: \Gamma(\sigma)=\int_{0}^{\infty} \tau^{\sigma-1} e^{-\tau} d \tau$.

In [36], Andrić et al. introduced the generalized fractional integral operators as follows:

Definition 2. Let $\vartheta, \theta, \sigma, l, \mu, c \in \mathbb{C}, \Re(\theta), \Re(\sigma), \Re(l)>0, \Re(c)>\Re(\mu)>0$ with $p \geq 0$, $r>0$ and $0<q \leq r+\Re(\theta)$. Let $f \in L_{1}[a, b]$ and $x \in[a, b]$. Then the generalized fractional integral operators are defined by:

$$
\begin{aligned}
& \left(\xi_{\theta, \sigma, l, \vartheta, a^{+}}^{\mu, r, q, c} f\right)(x ; p)=\int_{a}^{x}(x-\tau)^{\sigma-1} E_{\theta, \sigma, l}^{\mu, r, q, c}\left(\vartheta(x-\tau)^{\theta} ; p\right) f(\tau) d \tau, \\
& \left(\xi_{\theta, \sigma, l, \vartheta, b^{-}}^{\mu, r, q, c} f\right)(x ; p)=\int_{u}^{b}(\tau-x)^{\sigma-1} E_{\theta, \sigma, l}^{\mu, r, q, c}\left(\vartheta(\tau-x)^{\theta} ; p\right) f(\tau) d \tau,
\end{aligned}
$$

where $E_{\theta, \sigma, l}^{\mu, r, q, c}(\tau ; p)$ is the generalized Mittag-Leffler function defined by:

$$
\begin{gathered}
E_{\theta, \sigma, l}^{\mu, r, q, c}(\tau ; p)=\sum_{n=0}^{\infty} \frac{\mathrm{B}_{p}(\mu+n q, c-\mu)}{\mathrm{B}(\mu, c-\mu)} \frac{(c)_{n q}}{\Gamma(\theta n+\sigma)} \frac{\tau^{n}}{(l)_{n r}} \\
\mathrm{~B}_{p}(x, y)=\int_{0}^{1} \tau^{x-1}(1-\tau)^{y-1} e^{-\frac{p}{\tau(1-\tau)}} d \tau \text { and }(c)_{n q}=\frac{\Gamma(c+n q)}{\Gamma(c)}
\end{gathered}
$$

In [32], Farid introduced the unified integral operators as follows:

Definition 3. Let $f, \alpha:[a, b] \rightarrow \mathbb{R}, 0<a<b$ be the functions such that $f$ be a positive and integrable and $\alpha$ be a differentiable and strictly increasing. Also, let $\frac{\varphi}{x}$ be an increasing function on $[a, \infty)$ and $\sigma, l, \mu, c \in \mathbb{C}, p, \theta, r \geq 0$ and $0<q \leq r+\theta$. Then for $x \in[a, b]$ the integral operators are defined by:

$$
\begin{aligned}
& \left(\alpha \xi_{\theta, \sigma, l, \vartheta, a^{+}}^{\varphi ; \mu, r, q, c} f\right)(x ; p)=\int_{a}^{x} \frac{\varphi(\alpha(x)-\alpha(\tau))}{\alpha(x)-\alpha(\tau)} E_{\theta, \sigma, l}^{\mu, r, q, c}\left(\vartheta(\alpha(x)-\alpha(\tau))^{\theta} ; p\right) f(\tau) d(\alpha(\tau)), \\
& \left(\alpha \xi_{\theta, \sigma, l, \vartheta, b^{-}}^{\varphi ; \mu, r, q, c}\right)(x ; p)=\int_{x}^{b} \frac{\varphi(\alpha(\tau)-\alpha(x))}{\alpha(\tau)-\alpha(x)} E_{\theta, \sigma, l}^{\mu, r, q, c}\left(\vartheta(\alpha(\tau)-\alpha(x))^{\theta} ; p\right) f(\tau) d(\alpha(\tau)) .
\end{aligned}
$$

The following generalized $k$-integral operators involving Mittag-Leffler function with some modification is produced, for $\varphi(x)=x^{\frac{\sigma}{k}}$ with $k>0$, in (6) and (7):

Definition 4. Let $f, \alpha:[a, b] \rightarrow \mathbb{R}, 0<a<b$ be the functions such that $f$ be a positive and integrable and $\alpha$ be a differentiable and strictly increasing. Also, let $\theta, \sigma, l, \vartheta, \mu, c \in \mathbb{C}, p, \theta, r \geq 0$, $0<q \leq r+\theta$ and $k>0$. Then for $x \in[a, b]$ the integral operators are defined by:

$$
\begin{aligned}
& \left({ }_{\alpha}^{k} \xi_{\theta, \sigma, l, \vartheta, a^{+}}^{\mu, r, q, c} f\right)(x ; p)=\int_{a}^{x}(\alpha(x)-\alpha(\tau))^{\frac{\sigma}{k}-1} E_{\theta, \sigma, l, k}^{\mu, r, q, c}\left(\vartheta(\alpha(x)-\alpha(\tau))^{\frac{\theta}{k}} ; p\right) f(\tau) d(\alpha(\tau)),
\end{aligned}
$$

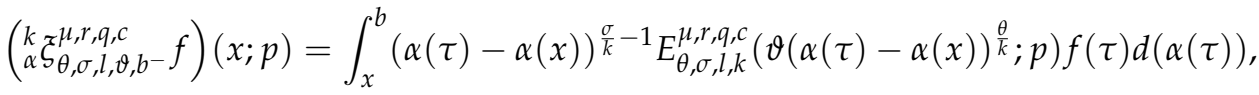


where $E_{\theta, \sigma, l, k}^{\mu, r, q, c}(\tau ; p)$ is the Mittag-Leffler function defined by:

$$
E_{\theta, \sigma, l, k}^{\mu, r, q, c}(\tau ; p)=\sum_{n=0}^{\infty} \frac{\mathrm{B}_{p}(\mu+n q, c-\mu)}{\mathrm{B}(\mu, c-\mu)} \frac{(c)_{n q}}{k \Gamma_{k}(\theta n+\sigma)} \frac{\tau^{n}}{(l)_{n r}} .
$$

Remark 1. The following integral operators can be deduced from (8) and (9):

1. The following integral operator is produced, for $\alpha(x)=x$ in (8):

$$
\left({ }^{k} \xi_{\theta, \sigma, l, \vartheta, a^{+}}^{\mu, r, q, c} f\right)(x ; p)=\int_{a}^{x}(x-\tau)^{\frac{\sigma}{k}-1} E_{\theta, \sigma, l, k}^{\mu, r, q, c}\left(\vartheta(x-\tau)^{\frac{\theta}{k}} ; p\right) f(\tau) d \tau .
$$

2. The following generalized Hadamard integral operator is produced, for $\alpha(x)=\ln x$ in (8):

$$
\left({ }^{k} \xi_{\theta, \sigma, l, \vartheta, a^{+}}^{\mu, r, q, c} f\right)(x ; p)=\int_{a}^{x}\left(\ln \frac{x}{\tau}\right)^{\frac{\sigma}{k}-1} E_{\theta, \sigma, l, k}^{\mu, r, q, c}\left(\vartheta\left(\ln \frac{x}{\tau}\right)^{\frac{\theta}{k}} ; p\right) f(\tau) \frac{d \tau}{\tau} .
$$

3. The following generalized Katugampola integral operator is produced, for $\alpha(x)=\frac{x^{\rho}}{\rho}, \rho>0$ in (8):

$$
\left({ }_{\rho}^{k} \xi_{\theta, \sigma, l, \vartheta, a^{+}}^{\mu, r, q, c} f\right)(x ; p)=\int_{a}^{x}\left(\frac{x^{\rho}-\tau^{\rho}}{\rho}\right)^{\frac{\sigma}{k}-1} E_{\theta, \sigma, l, k}^{\mu, r, q, c}\left(\vartheta\left(\frac{x^{\rho}-\tau^{\rho}}{\rho}\right)^{\frac{\theta}{k}} ; p\right) f(\tau) \tau^{\rho-1} d \tau .
$$

4. The following generalized ( $k, s)$-integral operator is produced, for $\alpha(x)=\frac{x^{s+1}}{s+1}, s \in \mathbb{R}-$ $\{-1\}$ in (8):

$$
\left({ }_{s}^{k} \xi_{\theta, \sigma, l, \vartheta, a^{+}}^{\mu, r, q, c} f\right)(x ; p)=\int_{a}^{x}\left(\frac{x^{s+1}-\tau^{s+1}}{s+1}\right)^{\frac{\sigma}{k}-1} E_{\theta, \sigma, l, k}^{\mu, r, q, c}\left(\vartheta\left(\frac{x^{s+1}-\tau^{s+1}}{s+1}\right)^{\frac{\theta}{k}} ; p\right) f(\tau) \tau^{s} d \tau .
$$

5. The following generalized conformable k-integral operator is produced, for $\alpha(x)=\frac{x^{\lambda+v}}{\lambda+v}$ in (8):

$$
\left({ }_{\lambda, \nu}^{k} \xi_{\theta, \sigma, l, l, a^{+}}^{\mu, r, q, c} f\right)(x ; p)=\int_{a}^{x}\left(\frac{x^{\lambda+v}-\tau^{\lambda+v}}{\lambda+v}\right)^{\frac{\sigma}{k}-1} E_{\theta, \sigma, l, k}^{\mu, r, q, c}\left(\vartheta\left(\frac{x^{\lambda+v}-\tau^{\lambda+v}}{\lambda+v}\right)^{\frac{\theta}{k}} ; p\right) f(\tau) \tau^{\lambda} d_{v} \tau .
$$

Similarly, all above operators can be deduce for generalized $k$-integral operators (9).

6. The following generalized conformable $(k, s)$-integral operators are produced, for $\alpha(x)=$ $\frac{(x-a)^{s}}{s}, s>0$ in (8) and $\alpha(x)=\frac{-(b-x)^{s}}{s}, s>0$ in (9):

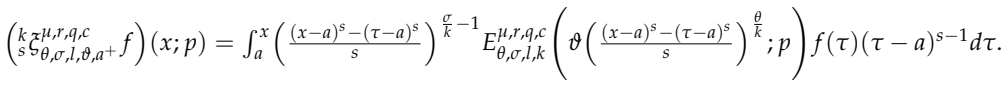

$$
\begin{aligned}
& \left({ }_{s}^{k} \mathcal{S}_{\theta, \sigma, l, \theta, b^{-}}^{\mu, r, c} f\right)(x ; p)=\int_{x}^{b}\left(\frac{(b-x)^{s}-(b-\tau)^{s}}{s}\right)^{\frac{\sigma}{k}-1} E_{\theta, \sigma, l, k}^{\mu, r, q, c}\left(\vartheta\left(\frac{(b-x)^{s}-(b-\tau)^{s}}{s}\right)^{\frac{\theta}{k}} ; p\right) f(\tau)(b-\tau)^{s-1} d \tau .
\end{aligned}
$$

Remark 2. For different choices of parameters involving in the Mittag-Leffler function (10), one can obtain new generalized integral operators.

Remark 3. From integral operators (8) and (9), we have the following particular cases:

1. The integral operators given in [29] are reproduced, for $k=1$.

2. The integral operators given in (4) and (5) are reproduced, for $k=1$ and $\alpha(x)=x$.

3. The integral operators given in [37] are reproduced, for $k=1, \alpha(x)=x$, and $p=0$.

4. The integral operators given in [38] are reproduced, for $k=1, \alpha(x)=x$, and $r=l=1$.

5. The integral operators given in [39] are reproduced, for $k=1, \alpha(x)=x, p=0$, and $r=l=1$.

6. The integral operators given in [40] are reproduced, for $k=1, \alpha(x)=x, p=0$, and $q=r=l=1$. 
7. The integral operators given in [26] are reproduced, for $k=1, \alpha(x)=\frac{x^{\rho}}{\rho}, \rho>0$, and $\vartheta=p=0$.

8. The integral operators given in [41] are reproduced, for $k=1, \alpha(x)=\ln x$, and $\vartheta=p=0$.

9. The integral operators given in [42] are reproduced, for $\alpha(x)=\frac{x^{s+1}}{s+1}$ and $\vartheta=p=0$.

10. The integral operators given in [30] are reproduced, for $k=1, \alpha(x)=\frac{(x)^{\lambda+v}}{\lambda+v}$ and $\vartheta=p=0$.

11. The integral operators given in [27] are reproduced, for $\alpha(x)=\frac{(x-a)^{s}}{s}, s>0$ in (8) and $\alpha(x)=-\frac{(b-x)^{s}}{s}, s>0$ in (9) with $\vartheta=p=0$.

12. The integral operators given in [33] are reproduced, for $\alpha(x)=\frac{(x-a)^{s}}{s}, s>0$ in (8) and $\alpha(x)=-\frac{(b-x)^{s}}{s}, s>0$ in (9) with $k=1$ and $\vartheta=p=0$.

13. The integral operators given in [28] are reproduced, for $\vartheta=p=0$.

14. The integral operators given in [41] are reproduced, for $\vartheta=p=0$ and $k=1$.

15. The integral operators given in [43] are reproduced, for $\vartheta=p=0$, and $\alpha(x)=x$.

16. The integral operators given in (2) and (3) are reproduced, for $\vartheta=p=0, \alpha(x)=x$, and $k=1$.

For constant function, from generalized $k$-fractional integral operator (8), we have

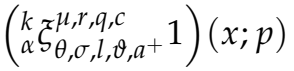

$$
\begin{aligned}
& =\int_{a}^{x}(\alpha(x)-\alpha(\tau))^{\frac{\sigma}{k}-1} E_{\theta, \sigma, l, k}^{\mu, r, q, c}\left(\vartheta(\alpha(x)-\alpha(\tau))^{\frac{\theta}{k}} ; p\right) d(\alpha(\tau)) \\
& =\int_{a}^{x}(\alpha(x)-\alpha(\tau))^{\frac{\sigma}{k}-1} \sum_{n=0}^{\infty} \frac{\mathrm{B}_{p}(\mu+n q, c-\mu)}{\mathrm{B}(\mu, c-\mu)} \frac{(c)_{n q}}{k \Gamma_{k}(\theta n+\sigma)} \frac{\vartheta^{n}(\alpha(x)-\alpha(\tau))^{\frac{\theta n}{k}}}{(l)_{n r}} d(\alpha(\tau)) \\
& =\sum_{n=0}^{\infty} \frac{\mathrm{B}_{p}(\mu+n q, c-\mu)}{\mathrm{B}(\mu, c-\mu)} \frac{(c)_{n q}}{k \Gamma_{k}(\theta n+\sigma)} \frac{\vartheta^{n}}{(l)_{n r}} \int_{a}^{x}(\alpha(x)-\alpha(\tau))^{\frac{\theta n}{k}+\frac{\sigma}{k}-1} d(\alpha(\tau)) \\
& =k(\alpha(x)-\alpha(a))^{\frac{\sigma}{k}} \sum_{n=0}^{\infty} \frac{\mathrm{B}_{p}(\mu+n q, c-\mu)}{\mathrm{B}(\mu, c-\mu)} \frac{(c)_{n q}}{k \Gamma_{k}(\theta n+\sigma)} \frac{\vartheta^{n}}{(l)_{n r}} \frac{(\alpha(x)-\alpha(a))^{\frac{\theta n}{k}}}{\theta n+\sigma} \\
& =k(\alpha(x)-\alpha(a))^{\frac{\sigma}{k}} \sum_{n=0}^{\infty} \frac{\mathrm{B}_{p}(\mu+n q, c-\mu)}{\mathrm{B}(\mu, c-\mu)} \frac{(c)_{n q}}{k \Gamma_{k}(\theta n+\sigma+k)} \frac{\vartheta^{n}(\alpha(x)-\alpha(a))^{\frac{\theta n}{k}}}{(l)_{n r}} \\
& =k(\alpha(x)-\alpha(a))^{\frac{\sigma}{k}} E_{\theta, \sigma+k, l, k}^{\mu, r, q, c}\left(\vartheta(\alpha(x)-\alpha(a))^{\frac{\theta}{k}} ; p\right) \text {. }
\end{aligned}
$$

Hence

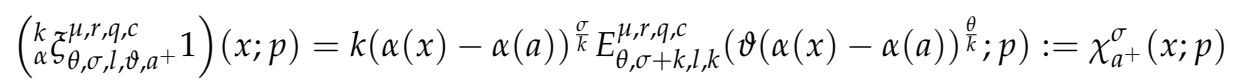

similarly for constant function, from generalized fractional integral operator (9), we get

$$
\left({ }_{\alpha}^{k} \xi_{\theta, \sigma, l, \vartheta, b^{-}}^{\mu, r, q, c} 1\right)(x ; p)=k(\alpha(b)-\alpha(x))^{\frac{\sigma}{k}} E_{\theta, \sigma+k, l, k}^{\mu, r, q, c}\left(\vartheta(\alpha(b)-\alpha(x))^{\frac{\theta}{k}} ; p\right):=\chi_{b^{-}}^{\sigma}(x ; p) .
$$

In the all upcoming results, the parameters of the Mittag-Leffler function are considered in $\mathbb{R}$.

\section{Pólya-Szegö and Chebyshev Type Inequalities for Generalized $k$-Fractional Integral Operators}

In this section, we obtain Pólya-Szegö and Chebyshev type inequalities for generalized $k$-fractional integral operators containing Mittag-Leffler function in their kernels. For the reader's convenience we will use a simplified notation:

$$
\begin{aligned}
& \left({ }_{\alpha}^{k} \mathbf{Z}_{\sigma} f\right)(x ; p):=\left({ }_{k}^{k} \xi_{\theta, \sigma, l, \vartheta, a^{+}}^{\mu, r, q, c} f\right)(x ; p) \\
& =\int_{a}^{x}(\alpha(x)-\alpha(\tau))^{\frac{\sigma}{k}-1} \mathbf{E}_{\sigma}^{k}\left(\vartheta(\alpha(x)-\alpha(\tau))^{\frac{\theta}{k}} ; p\right) f(\tau) d(\alpha(\tau)),
\end{aligned}
$$


where

$$
\begin{aligned}
\mathbf{E}_{\sigma}^{k}:= & E_{\theta, \sigma, l, k}^{\mu, r, q}(\tau ; p) \\
& =\sum_{n=0}^{\infty} \frac{\mathrm{B}_{p}(\mu+n q, c-\mu)}{\mathrm{B}(\mu, c-\mu)} \frac{(c)_{n q}}{k \Gamma_{k}(\theta n+\sigma)} \frac{\tau^{n}}{(l)_{n r}} .
\end{aligned}
$$

Theorem 1. Suppose that:

- $\quad f$ and $g$ be two positive and integrable functions on $[0, \infty)$;

- $\quad \alpha:[a, b] \rightarrow \mathbb{R}$ be an increasing and differentiable function with $\alpha^{\prime} \in L[a, b] ;$

- there exist four positive integrable functions $\zeta_{1}, \zeta_{2}, \eta_{1}$ and $\eta_{2}$, such that

$$
0<\zeta_{1}(\tau) \leq f(\tau) \leq \zeta_{2}(\tau), \quad 0<\eta_{1}(\tau) \leq g(\tau) \leq \eta_{2}(\tau) \quad(\tau \in[a, x], x>a) .
$$

Then for generalized $k$-fractional integral operator containing Mittag-Leffler function, we have

$$
\frac{\left({ }_{\alpha}^{k} Z_{\sigma} \eta_{1} \eta_{2} f^{2}\right)(x ; p)\left({ }_{\alpha}^{k} Z_{\sigma} \zeta_{1} \zeta_{2} g^{2}\right)(x ; p)}{\left[\left({ }_{\alpha}^{k} Z_{\sigma}\left(\zeta_{1} \eta_{1}+\zeta_{2} \eta_{2}\right) f g\right)(x ; p)\right]^{2}} \leq \frac{1}{4} .
$$

Proof. From (20) for $\tau \in[a, x]$ with $x>a$, we can write

$$
\left(\frac{\zeta_{2}(\tau)}{\eta_{1}(\tau)}-\frac{f(\tau)}{g(\tau)}\right)\left(\frac{f(\tau)}{g(\tau)}-\frac{\zeta_{1}(\tau)}{\eta_{2}(\tau)}\right) \geq 0,
$$

which implies

$$
\left(\zeta_{1}(\tau) \eta_{1}(\tau)+\zeta_{2}(\tau) \eta_{2}(\tau)\right) f(\tau) g(\tau) \geq \eta_{1}(\tau) \eta_{2}(\tau) f^{2}(\tau)+\zeta_{1}(\tau) \zeta_{2}(\tau) g^{2}(\tau) .
$$

Multiplying (22) with $(\alpha(x)-\alpha(\tau))^{\frac{\sigma}{k}-1} \mathbf{E}_{\sigma}^{k}\left(\vartheta(\alpha(x)-\alpha(\tau))^{\theta} ; p\right) \alpha^{\prime}(\tau)$ on both sides and integrating, we get

$$
\begin{aligned}
& \int_{a}^{x}(\alpha(x)-\alpha(\tau))^{\frac{\sigma}{k}-1} \mathbf{E}_{\sigma}^{k}\left(\vartheta(\alpha(x)-\alpha(\tau))^{\theta} ; p\right)\left(\zeta_{1}(\tau) \eta_{1}(\tau)+\zeta_{2}(\tau) \eta_{2}(\tau)\right) f(\tau) g(\tau) \alpha^{\prime}(\tau) d \tau \\
& \geq \int_{a}^{x}(\alpha(x)-\alpha(\tau))^{\frac{\sigma}{k}-1} \mathbf{E}_{\sigma}^{k}\left(\vartheta(\alpha(x)-\alpha(\tau))^{\theta} ; p\right) \eta_{1}(\tau) \eta_{2}(\tau) f^{2}(\tau) \alpha^{\prime}(\tau) d \tau \\
& +\int_{a}^{x}(\alpha(x)-\alpha(\tau))^{\frac{\sigma}{k}-1} \mathbf{E}_{\sigma}^{k}\left(\vartheta(\alpha(x)-\alpha(\tau))^{\theta} ; p\right) \zeta_{1}(\tau) \zeta_{2}(\tau) g^{2}(\tau) \alpha^{\prime}(\tau) d \tau .
\end{aligned}
$$

Now by using $k$-fractional integral operator, we get

$$
\left({ }_{\alpha}^{k} \mathbf{Z}_{\sigma}\left(\zeta_{1} \eta_{1}+\zeta_{2} \eta_{2}\right) f g\right)(x ; p) \geq\left({ }_{\alpha}^{k} \mathbf{Z}_{\sigma} \eta_{1} \eta_{2} f^{2}\right)(x ; p)+\left({ }_{\alpha}^{k} \mathbf{Z}_{\sigma} \zeta_{1} \zeta_{2} g^{2}\right)(x ; p) .
$$

By applying AM-GM inequality, we get

$$
\left({ }_{\alpha}^{k} \mathbf{Z}_{\sigma}\left(\zeta_{1} \eta_{1}+\zeta_{2} \eta_{2}\right) f g\right)(x ; p) \geq 2 \sqrt{\left({ }_{\alpha}^{k} \mathbf{Z}_{\sigma} \eta_{1} \eta_{2} f^{2}\right)(x ; p)\left({ }_{\alpha}^{k} \mathbf{Z}_{\sigma} \zeta_{1} \zeta_{2} g^{2}\right)(x ; p)},
$$

which leads to the required inequality (21).

Corollary 1. If $\zeta_{1}=u, \zeta_{2}=U, \eta_{1}=v$ and $\eta_{2}=V$, then we have

$$
\frac{\left({ }_{\alpha}^{k} Z_{\sigma} f^{2}\right)(x ; p)\left({ }_{\alpha}^{k} Z_{\sigma} g^{2}\right)(x ; p)}{\left[\left({ }_{\alpha}^{k} Z_{\sigma} f g\right)(x ; p)\right]^{2}} \leq \frac{1}{4}\left(\sqrt{\frac{u v}{U V}}+\sqrt{\frac{U V}{u v}}\right)^{2} .
$$

Remark 4. In Theorem 1, for $\alpha(x)=x$ and $k=1$, we get [14] (Theorem 1), for $\vartheta=p=0$ (and $a=0$ ), we get [19] (Lemma 3.1), for $\alpha(x)=x, k=1, \vartheta=p=0$ (and $a=0$ ), we get [10] (Lemma 3.1). 
Theorem 2. Under the assumptions of Theorem 1 with $\varsigma>0$, we have

$$
\frac{\left({ }_{\alpha}^{k} \boldsymbol{Z}_{\sigma} \zeta_{1} \zeta_{2}\right)(x ; p)\left({ }_{\alpha}^{k} \boldsymbol{Z}_{\zeta} \eta_{1} \eta_{2}\right)(x ; p)\left({ }_{\alpha}^{k} \boldsymbol{Z}_{\sigma} f^{2}\right)(x ; p)\left({ }_{\alpha}^{k} \boldsymbol{Z}_{\zeta} g^{2}\right)(x ; p)}{\left[\left({ }_{\alpha}^{k} \boldsymbol{Z}_{\sigma} \zeta_{1} f\right)(x ; p)\left({ }_{\alpha}^{k} \boldsymbol{Z}_{\zeta} \eta_{1} g\right)(x ; p)+\left({ }_{\alpha}^{k} \boldsymbol{Z}_{\sigma} \zeta_{2} f\right)(x ; p)\left({ }_{\alpha}^{k} \boldsymbol{Z}_{\zeta} \eta_{2} g\right)(x ; p)\right]^{2}} \leq \frac{1}{4} .
$$

Proof. From (20), for $\tau, \kappa \in[a, x]$ with $x>a$, we can write

$$
\left(\frac{\zeta_{1}(\tau)}{\eta_{2}(\kappa)}+\frac{\zeta_{2}(\tau)}{\eta_{1}(\kappa)}\right) \frac{f(\tau)}{g(\kappa)} \geq \frac{f^{2}(\tau)}{g^{2}(\kappa)}+\frac{\zeta_{1}(\tau) \zeta_{2}(\tau)}{\eta_{1}(\kappa) \eta_{2}(\kappa)}
$$

which imply

$$
\zeta_{1}(\tau) f(\tau) \eta_{1}(\kappa) g(\kappa)+\zeta_{2}(\tau) f(\tau) \eta_{2}(\kappa) g(\kappa) \geq \eta_{1}(\kappa) \eta_{2}(\kappa) f^{2}(\tau)+\zeta_{1}(\tau) \zeta_{2}(\tau) g^{2}(\kappa) .
$$

Multiplying (24) with $(\alpha(x)-\alpha(\tau))^{\frac{\sigma}{k}-1}(\alpha(x)-\alpha(\kappa))^{\frac{\zeta}{k}-1} \mathbf{E}_{\sigma}^{k}\left(\vartheta(\alpha(x)-\alpha(\tau))^{\theta} ; p\right)$ $\mathbf{E}_{\zeta}^{k}\left(\vartheta(\alpha(x)-\alpha(\kappa))^{\theta} ; p\right) \alpha^{\prime}(\tau) \alpha^{\prime}(\kappa)$ on both sides and integrating, we get

$$
\begin{aligned}
& \int_{a}^{x} \int_{a}^{x}(\alpha(x)-\alpha(\tau))^{\frac{\sigma}{k}-1}(\alpha(x)-\alpha(\kappa))^{\frac{\zeta}{k}-1} \mathbf{E}_{\sigma}^{k}\left(\vartheta(\alpha(x)-\alpha(\tau))^{\theta} ; p\right) \\
& \times \mathbf{E}_{\zeta}^{k}\left(\vartheta(\alpha(x)-\alpha(\kappa))^{\theta} ; p\right) \zeta_{1}(\tau) f(\tau) \eta_{1}(\kappa) g(\kappa) \alpha^{\prime}(\tau) \alpha^{\prime}(\kappa) d \tau d \kappa \\
& +\int_{a}^{x} \int_{a}^{x}(\alpha(x)-\alpha(\tau))^{\frac{\sigma}{k}-1}(\alpha(x)-\alpha(\kappa))^{\frac{\zeta}{k}-1} \mathbf{E}_{\sigma}^{k}\left(\vartheta(\alpha(x)-\alpha(\tau))^{\theta} ; p\right) \\
& \times \mathbf{E}_{\zeta}^{k}\left(\vartheta(\alpha(x)-\alpha(\kappa))^{\theta} ; p\right) \zeta_{2}(\tau) f(\tau) \eta_{2}(\kappa) g(\kappa) \alpha^{\prime}(\tau) \alpha^{\prime}(\kappa) d \tau d \kappa \\
& \geq \\
& \int_{a}^{x} \int_{a}^{x}(\alpha(x)-\alpha(\tau))^{\frac{\sigma}{k}-1}(\alpha(x)-\alpha(\kappa))^{\frac{\zeta}{k}-1} \mathbf{E}_{\sigma}^{k}\left(\vartheta(\alpha(x)-\alpha(\tau))^{\theta} ; p\right) \\
& \times \mathbf{E}_{\zeta}^{k}\left(\vartheta(\alpha(x)-\alpha(\kappa))^{\theta} ; p\right) \eta_{1}(\kappa) \eta_{2}(\kappa) f^{2}(\tau) \alpha^{\prime}(\tau) \alpha^{\prime}(\kappa) d \tau d \kappa \\
& +\int_{a}^{x} \int_{a}^{x}(\alpha(x)-\alpha(\tau))^{\frac{\sigma}{k}-1}(\alpha(x)-\alpha(\kappa))^{\frac{\zeta}{k}-1} \mathbf{E}_{\sigma}^{k}\left(\vartheta(\alpha(x)-\alpha(\tau))^{\theta} ; p\right) \\
& \times \mathbf{E}_{\zeta}^{k}\left(\vartheta(\alpha(x)-\alpha(\kappa))^{\theta} ; p\right) \zeta_{1}(\tau) \zeta_{2}(\tau) g^{2}(\kappa) \alpha^{\prime}(\tau) \alpha^{\prime}(\kappa) d \tau d \kappa .
\end{aligned}
$$

Now by using $k$-fractional integral operator, we get

$$
\begin{aligned}
& \left({ }_{\alpha}^{k} \mathbf{Z}_{\sigma} \zeta_{1} f\right)(x ; p)\left({ }_{\alpha}^{k} \mathbf{Z}_{\varsigma} \eta_{1} g\right)(x ; p)+\left({ }_{\alpha}^{k} \mathbf{Z}_{\sigma} \zeta_{2} f\right)(x ; p)\left({ }_{\alpha}^{k} \mathbf{Z}_{\varsigma} \eta_{2} g(x ; p)\right) \\
& \geq\left({ }_{\alpha}^{k} \mathbf{Z}_{\sigma} f^{2}\right)(x ; p)\left({ }_{\alpha}^{k} \mathbf{Z}_{\varsigma} \eta_{1} \eta_{2}\right)(x ; p)+\left({ }_{\alpha}^{k} \mathbf{Z}_{\sigma} \zeta_{1} \zeta_{2}\right)(x ; p)\left({ }_{\alpha}^{k} \mathbf{Z}_{\zeta} g^{2}\right)(x ; p) .
\end{aligned}
$$

By applying AM-GM inequality, we get

$$
\begin{aligned}
& \left({ }_{\alpha}^{k} \mathbf{Z}_{\sigma} \zeta_{1} f\right)(x ; p)\left({ }_{\alpha}^{k} \mathbf{Z}_{\zeta} \eta_{1} g\right)(x ; p)+\left({ }_{\alpha}^{k} \mathbf{Z}_{\sigma} \zeta_{2} f\right)(x ; p)\left({ }_{\alpha}^{k} \mathbf{Z}_{\zeta} \eta_{2} g\right)(x ; p) \\
& \geq 2 \sqrt{\left({ }_{\alpha}^{k} \mathbf{Z}_{\sigma} f^{2}\right)(x ; p)\left({ }_{\alpha}^{k} \mathbf{Z}_{\zeta} \eta_{1} \eta_{2}\right)(x ; p)\left({ }_{\alpha}^{k} \mathbf{Z}_{\sigma} \zeta_{1} \zeta_{2}\right)(x ; p)\left({ }_{\alpha}^{k} \mathbf{Z}_{\zeta} g^{2}\right)(x ; p)},
\end{aligned}
$$

which leads to the required inequality (23).

Corollary 2. If $\zeta_{1}=u, \zeta_{2}=U, \eta_{1}=v$ and $\eta_{2}=V$, then we have

$$
\frac{\chi_{\sigma}(x ; p) \chi_{\varsigma}(x ; p)\left({ }_{\alpha}^{k} Z_{\sigma} f^{2}\right)(x ; p)\left({ }_{\alpha}^{k} Z_{\zeta} g^{2}\right)(x ; p)}{\left[\left({ }_{\alpha} Z_{\sigma} f\right)(x ; p)\left({ }_{\alpha}^{k} Z_{\zeta} g\right)(x ; p)\right]^{2}} \leq \frac{1}{4}\left(\sqrt{\frac{u v}{U V}}+\sqrt{\frac{U V}{u v}}\right)^{2} .
$$

Remark 5. In Theorem 2, for $\alpha(x)=x$ and $k=1$, we get [14] (Theorem 2), for $\vartheta=p=0$ (and $a=0$ ), we get [19] (Lemma 3.6), for $\alpha(x)=x, k=1, \vartheta=p=0$ (and $a=0$ ), we get [10] (Lemma 3.3). 
Theorem 3. Under the assumptions of Theorem 1 with $\varsigma>0$, we have

$$
\left({ }_{\alpha}^{k} Z_{\sigma} f^{2}\right)(x ; p)\left({ }_{\alpha}^{k} Z_{\zeta} g^{2}\right)(x ; p) \leq\left({ }_{\alpha}^{k} Z_{\sigma}\left(\zeta_{2} f g / \eta_{1}\right)\right)(x ; p)\left({ }_{\alpha}^{k} Z_{\zeta}\left(\eta_{2} f g / \zeta_{1}\right)\right)(x ; p) .
$$

Proof. From (20), for $\tau \in[a, x]$ with $x>a$, we can write

$$
\frac{\zeta_{2}(\tau) f(\tau) g(\tau)}{\eta_{1}(\tau)}-f^{2}(\tau) \geq 0
$$

and

$$
\frac{\eta_{2}(\kappa) f(\kappa) g(\kappa)}{\zeta_{1}(\kappa)}-g^{2}(\kappa) \geq 0 .
$$

Multiplying (26) with $(\alpha(x)-\alpha(\tau))^{\frac{\sigma}{k}-1} \mathbf{E}_{\sigma}^{k}\left(\vartheta(\alpha(x)-\alpha(\tau))^{\theta} ; p\right) \alpha^{\prime}(\tau)$ and (27) with $(\alpha(x)-\alpha(\kappa))^{\frac{\zeta}{k}-1} \mathbf{E}_{\zeta}^{k}\left(\vartheta(\alpha(x)-\alpha(\kappa))^{\theta} ; p\right) \alpha^{\prime}(\kappa)$ on both sides and integrating, we get

$$
\begin{aligned}
& \int_{a}^{x}(\alpha(x)-\alpha(\tau))^{\frac{\sigma}{k}-1} \mathbf{E}_{\sigma}^{k}\left(\vartheta(\alpha(x)-\alpha(\tau))^{\theta} ; p\right) f^{2}(\tau) \alpha^{\prime}(\tau) d \tau \\
& \leq \int_{a}^{x}(\alpha(x)-\alpha(\tau))^{\frac{\sigma}{k}-1} \mathbf{E}_{\sigma}^{k}\left(\vartheta(\alpha(x)-\alpha(\tau))^{\theta} ; p\right) \frac{\zeta_{2}(\tau)}{\eta_{1}(\tau)} f(\tau) g(\tau) \alpha^{\prime}(\tau) d \tau
\end{aligned}
$$

and

$$
\begin{aligned}
& \int_{a}^{x}(\alpha(x)-\alpha(\kappa))^{\frac{\varsigma}{k}-1} \mathbf{E}_{\mathcal{\zeta}}^{k}\left(\vartheta(\alpha(x)-\alpha(\kappa))^{\theta} ; p\right) g^{2}(\kappa) \alpha^{\prime}(\kappa) d \kappa \\
& \leq \int_{a}^{x}(\alpha(x)-\alpha(\kappa))^{\frac{\varsigma}{k}-1} \mathbf{E}_{\mathcal{\zeta}}^{k}\left(\vartheta(\alpha(x)-\alpha(\kappa))^{\theta} ; p\right) \frac{\eta_{2}(\kappa)}{\zeta_{1}(\kappa)} f(\kappa) g(\kappa) \alpha^{\prime}(\kappa) d \kappa .
\end{aligned}
$$

Now by using $k$-fractional integral operator, we get

$$
\left({ }_{\alpha}^{k} \mathbf{Z}_{\sigma} f^{2}\right)(x ; p) \leq\left({ }_{\alpha}^{k} \mathbf{Z}_{\sigma}\left(\zeta_{2} f g / \eta_{1}\right)\right)(x ; p)
$$

and

$$
\left({ }_{\alpha}^{k} \mathbf{Z}_{\zeta} g^{2}\right)(x ; p) \leq\left({ }_{\alpha}^{k} \mathbf{Z}_{\zeta}\left(\eta_{2} f g / \zeta_{1}\right)\right)(x ; p)
$$

Multiplying (28) with (29), we obtain (25).

Corollary 3. If $\zeta_{1}=u, \zeta_{2}=U, \eta_{1}=v$ and $\eta_{2}=V$, then we have

$$
\frac{\left({ }_{\alpha}^{k} Z_{\sigma} f^{2}\right)(x ; p)\left({ }_{\alpha}^{k} Z_{\zeta} g^{2}\right)(x ; p)}{\left({ }_{\alpha}^{k} Z_{\sigma} f g\right)(x ; p)\left({ }_{\alpha}^{k} Z_{\zeta} f g\right)(x ; p)} \leq \frac{U V}{u v} .
$$

Remark 6. In Theorem 3, for $\alpha(x)=x$ and $k=1$, we get [14] (Theorem 3), for $\alpha(x)=x, k=1$, $\vartheta=p=0$ (and $a=0)$, we get [10] (Lemma 3.4).

The Chebyshev type inequalities for generalized $k$-fractional integral operators are given as follows:

Theorem 4. Under the assumptions of Theorem 1 with $\varsigma>0$, we have

$$
\begin{array}{r}
\mid \chi_{\sigma}(x ; p)\left({ }_{\alpha}^{k} Z_{\zeta} f g\right)(x ; p)+\chi_{\zeta}(x ; p)\left({ }_{\alpha}^{k} Z_{\sigma} f g\right)(x ; p) \\
\leq \quad-\left({ }_{\alpha}^{k} Z_{\sigma} f\right)(x ; p)\left({ }_{\alpha}^{k} Z_{\zeta} g\right)(x ; p)-\left({ }_{\alpha}^{k} Z_{\sigma} g\right)(x ; p)\left({ }_{\alpha}^{k} Z_{\zeta} f\right)(x ; p) \mid \\
\quad\left|G_{\sigma, \zeta}\left(f, \zeta_{1}, \zeta_{2}\right)(x ; p)+G_{\zeta, \sigma}\left(f, \zeta_{1}, \zeta_{2}\right)(x ; p)\right|^{\frac{1}{2}} \\
\times\left|G_{\sigma, \zeta}\left(g, \eta_{1}, \eta_{2}\right)(x ; p)+G_{\zeta, \sigma}\left(g, \eta_{1}, \eta_{2}\right)(x ; p)\right|^{\frac{1}{2}},
\end{array}
$$


where

$$
G_{\sigma, \zeta}(m, n, o)(x ; p)=\frac{\chi_{\varsigma}(x ; p)\left[\left({ }_{\alpha}^{k} \boldsymbol{Z}_{\sigma}(n+o) m\right)(x ; p)\right]^{2}}{4\left({ }_{\alpha}^{k} \mathbf{Z}_{\sigma} n o\right)(x ; p)}
$$

Proof. Let $f$ and $g$ be two positive and integrable functions on $[0, \infty)$. For $\tau, \kappa \in[a, x]$ with $x>a$, we define $A(\tau, \kappa)$ as

$$
A(\tau, \kappa)=(f(\tau)-f(\kappa))(g(\tau)-g(\kappa)),
$$

which imply

$$
A(\tau, \kappa)=f(\tau) g(\tau)+f(\kappa) g(\kappa)-f(\tau) g(\kappa)-f(\kappa) g(\tau) .
$$

Multiplying (32) with $(\alpha(x)-\alpha(\tau))^{\frac{\sigma}{k}-1}(\alpha(x)-\alpha(\kappa))^{\frac{\zeta}{k}-1} \mathbf{E}_{\sigma}^{k}\left(\vartheta(\alpha(x)-\alpha(\tau))^{\theta} ; p\right)$ $\mathbf{E}_{\zeta}^{k}\left(\vartheta(\alpha(x)-\alpha(\kappa))^{\theta} ; p\right) \alpha^{\prime}(\tau) \alpha^{\prime}(\kappa)$ and integrating, we get

$$
\begin{aligned}
\int_{a}^{x} \int_{a}^{x}(\alpha(x)-\alpha(\tau))^{\frac{\sigma}{k}-1}(\alpha(x)-\alpha(\kappa))^{\frac{\zeta}{k}-1} \mathbf{E}_{\sigma}^{k}\left(\vartheta(\alpha(x)-\alpha(\tau))^{\theta} ; p\right) \\
\times \mathbf{E}_{\zeta}^{k}\left(\vartheta(\alpha(x)-\alpha(\kappa))^{\theta} ; p\right) A(\tau, \kappa) \alpha^{\prime}(\tau) \alpha^{\prime}(\kappa) d \tau d \kappa
\end{aligned}
$$

Now by using $k$-fractional integral operator, we get

$$
\begin{aligned}
& \chi_{\varsigma}(x ; p)\left({ }_{\alpha}^{k} \mathbf{Z}_{\sigma} f g\right)(x ; p)+\chi_{\sigma}(x ; p)\left({ }_{\alpha}^{k} \mathbf{Z}_{\zeta} f g\right)(x ; p) \\
& -\left({ }_{\alpha}^{k} \mathbf{Z}_{\sigma} f\right)(x ; p)\left({ }_{\alpha}^{k} \mathbf{Z}_{\zeta} g\right)(x ; p)-\left({ }_{\alpha}^{k} \mathbf{Z}_{\zeta} f\right)(x ; p)\left({ }_{\alpha}^{k} \mathbf{Z}_{\sigma} g\right)(x ; p) .
\end{aligned}
$$

By using Cauchy-Schwartz inequality, we have

$$
\begin{aligned}
& \mid \int_{a}^{x} \int_{a}^{x}(\alpha(x)-\alpha(\tau))^{\frac{\sigma}{k}-1}(\alpha(x)-\alpha(\kappa))^{\frac{\zeta}{k}-1} \mathbf{E}_{\sigma}^{k}\left(\vartheta(\alpha(x)-\alpha(\tau))^{\theta} ; p\right) \\
& \times \mathbf{E}_{\zeta}^{k}\left(\vartheta(\alpha(x)-\alpha(\kappa))^{\theta} ; p\right) A(\tau, \kappa) \alpha^{\prime}(\tau) \alpha^{\prime}(\kappa) d \tau d \kappa \mid \\
& \leq\left[\int_{a}^{x} \int_{a}^{x}(\alpha(x)-\alpha(\tau))^{\frac{\sigma}{k}-1}(\alpha(x)-\alpha(\kappa))^{\frac{\zeta}{k}-1} \mathbf{E}_{\sigma}^{k}\left(\vartheta(\alpha(x)-\alpha(\tau))^{\theta} ; p\right)\right. \\
& \times \mathbf{E}_{\zeta}^{k}\left(\vartheta(\alpha(x)-\alpha(\kappa))^{\theta} ; p\right) f^{2}(\tau) \alpha^{\prime}(\tau) \alpha^{\prime}(\kappa) d \tau d \kappa \\
& +\int_{a}^{x} \int_{a}^{x}(\alpha(x)-\alpha(\tau))^{\frac{\sigma}{k}-1}(\alpha(x)-\alpha(\kappa))^{\frac{\zeta}{k}-1} \mathbf{E}_{\sigma}^{k}\left(\vartheta(\alpha(x)-\alpha(\tau))^{\theta} ; p\right) \\
& \times \mathbf{E}_{\zeta}^{k}\left(\vartheta(\alpha(x)-\alpha(\kappa))^{\theta} ; p\right) f^{2}(\kappa) \alpha^{\prime}(\tau) \alpha^{\prime}(\kappa) d \tau d \kappa \\
& -2 \int_{a}^{x} \int_{a}^{x}(\alpha(x)-\alpha(\tau))^{\frac{\sigma}{k}-1}(\alpha(x)-\alpha(\kappa))^{\frac{\zeta}{k}-1} \mathbf{E}_{\sigma}^{k}\left(\vartheta(\alpha(x)-\alpha(\tau))^{\theta} ; p\right) \\
& \left.\times \mathbf{E}_{\zeta}^{k}\left(\vartheta(\alpha(x)-\alpha(\kappa))^{\theta} ; p\right) f(\tau) f(\kappa) \alpha^{\prime}(\tau) \alpha^{\prime}(\kappa) d \tau d \kappa\right]^{\frac{1}{2}} \\
& \times\left[\int_{a}^{x} \int_{a}^{x}(\alpha(x)-\alpha(\tau))^{\frac{\sigma}{k}-1}(\alpha(x)-\alpha(\kappa))^{\frac{\zeta}{k}-1} \mathbf{E}_{\sigma}^{k}\left(\vartheta(\alpha(x)-\alpha(\tau))^{\theta} ; p\right)\right. \\
& \times \mathbf{E}_{\zeta}^{k}\left(\vartheta(\alpha(x)-\alpha(\kappa))^{\theta} ; p\right) g^{2}(\tau) \alpha^{\prime}(\tau) \alpha^{\prime}(\kappa) d \tau d \kappa \\
& +\int_{a}^{x} \int_{a}^{x}(\alpha(x)-\alpha(\tau))^{\frac{\sigma}{k}-1}(\alpha(x)-\alpha(\kappa))^{\frac{\zeta}{k}-1} \mathbf{E}_{\sigma}^{k}\left(\vartheta(\alpha(x)-\alpha(\tau))^{\theta} ; p\right) \\
& \times \mathbf{E}_{\zeta}^{k}\left(\vartheta(\alpha(x)-\alpha(\kappa))^{\theta} ; p\right) g^{2}(\kappa) \alpha^{\prime}(\tau) \alpha^{\prime}(\kappa) d \tau d \kappa
\end{aligned}
$$




$$
\begin{aligned}
& -2 \int_{a}^{x} \int_{a}^{x}(\alpha(x)-\alpha(\tau))^{\frac{\sigma}{k}-1}(\alpha(x)-\alpha(\kappa))^{\frac{\zeta}{k}-1} \mathbf{E}_{\sigma}^{k}\left(\vartheta(\alpha(x)-\alpha(\tau))^{\theta} ; p\right) \\
& \left.\times \mathbf{E}_{\zeta}^{k}\left(\vartheta(\alpha(x)-\alpha(\kappa))^{\theta} ; p\right) g(\tau) g(\kappa) \alpha^{\prime}(\tau) \alpha^{\prime}(\kappa) d \tau d \kappa\right]^{\frac{1}{2}} \\
& \leq\left[\chi_{\zeta}(x ; p)\left({ }_{\alpha}^{k} \mathbf{Z}_{\sigma} f^{2}\right)(x ; p)+\chi_{\sigma}(x ; p)\left({ }_{\alpha}^{k} \mathbf{Z}_{\zeta} f^{2}\right)(x ; p)-2\left({ }_{\alpha}^{k} \mathbf{Z}_{\sigma} f\right)(x ; p)\left({ }_{\alpha}^{k} \mathbf{Z}_{\zeta} f\right)(x ; p)\right]^{\frac{1}{2}} \\
& \times\left[\chi_{\zeta}(x ; p)\left({ }_{\alpha}^{k} \mathbf{Z}_{\sigma} g^{2}\right)(x ; p)+\chi_{\sigma}(x ; p)\left({ }_{\alpha}^{k} \mathbf{Z}_{\zeta} g^{2}\right)(x ; p)-2\left({ }_{\alpha}^{k} \mathbf{Z}_{\sigma} g\right)(x ; p)\left({ }_{\alpha}^{k} \mathbf{Z}_{\zeta} g\right)(x ; p)\right]^{\frac{1}{2}} .
\end{aligned}
$$

By taking $\eta_{1}(t)=\eta_{2}(t)=g(t)=1$ in Theorem 1 , we get the following inequality:

$$
\left({ }_{\alpha}^{k} \mathbf{Z}_{\sigma} f^{2}\right)(x ; p) \leq \frac{\left[\left({ }_{\alpha}^{k} \mathbf{Z}_{\sigma}\left(\zeta_{1}+\zeta_{2}\right) f\right)(x ; p)\right]^{2}}{4\left({ }_{\alpha}^{k} \mathbf{Z}_{\sigma} \zeta_{1} \zeta_{2}\right)(x ; p)} .
$$

This implies

$$
\begin{aligned}
& \chi_{\varsigma}(x ; p)\left({ }_{\alpha}^{k} \mathbf{Z}_{\sigma} f^{2}\right)(x ; p)-\left({ }_{\alpha}^{k} \mathbf{Z}_{\sigma} f\right)(x ; p)\left({ }_{\alpha}^{k} \mathbf{Z}_{\zeta} f\right)(x ; p) \\
& \leq \frac{\chi_{\varsigma}(x ; p)\left[\left({ }_{\alpha}^{k} \mathbf{Z}_{\sigma}\left(\zeta_{1}+\zeta_{2}\right) f\right)(x ; p)\right]^{2}}{4\left({ }_{\alpha}^{k} \mathbf{Z}_{\sigma} \zeta_{1} \zeta_{2}\right)(x ; p)}-\left({ }_{\alpha}^{k} \mathbf{Z}_{\sigma} f\right)(x ; p)\left({ }_{\alpha}^{k} \mathbf{Z}_{\zeta} f\right)(x ; p) \\
& =G_{\sigma, \zeta}\left(f, \zeta_{1}, \zeta_{2}\right)(x ; p)
\end{aligned}
$$

and

$$
\begin{aligned}
& \chi_{\sigma}(x ; p)\left({ }_{\alpha}^{k} \mathbf{Z}_{\zeta} f^{2}\right)(x ; p)-\left({ }_{\alpha}^{k} \mathbf{Z}_{\sigma} f\right)(x ; p)\left({ }_{\alpha}^{k} \mathbf{Z}_{\zeta} f\right)(x ; p) \\
& \leq \frac{\chi_{\sigma}(x ; p)\left[\left({ }_{\alpha}^{k} \mathbf{Z}_{\zeta}\left(\zeta_{1}+\zeta_{2}\right) f\right)(x ; p)\right]^{2}}{4\left({ }_{\alpha}^{k} \mathbf{Z}_{\zeta} \zeta_{1} \zeta_{2}\right)(x ; p)}-\left({ }_{\alpha}^{k} \mathbf{Z}_{\sigma} f\right)(x ; p)\left({ }_{\alpha}^{k} \mathbf{Z}_{\zeta} f\right)(x ; p) \\
& =G_{\zeta, \sigma}\left(f, \zeta_{1}, \zeta_{2}\right)(x ; p) .
\end{aligned}
$$

Applying the same procedure for $\zeta_{1}(t)=\zeta_{2}(t)=f(t)=1$, we get the following inequalities:

$$
\chi_{\varsigma}(x ; p)\left({ }_{\alpha}^{k} \mathbf{Z}_{\sigma} g^{2}\right)(x ; p)-\left({ }_{\alpha}^{k} \mathbf{Z}_{\sigma} g\right)(x ; p)\left({ }_{\alpha}^{k} \mathbf{Z}_{\zeta} g\right)(x ; p) \leq G_{\sigma, \zeta}\left(g, \eta_{1}, \eta_{2}\right)(x ; p)
$$

and

$$
\chi_{\sigma}(x ; p)\left({ }_{\alpha}^{k} \mathbf{Z}_{\zeta} g^{2}\right)(x ; p)-\left({ }_{\alpha}^{k} \mathbf{Z}_{\sigma} g\right)(x ; p)\left({ }_{\alpha}^{k} \mathbf{Z}_{\zeta} g\right)(x ; p) \leq G_{\zeta, \sigma}\left(g, \eta_{1}, \eta_{2}\right)(x ; p) .
$$

Finally, considering (33) to (37), we arrive at the desired result in (30).

Theorem 5. Under the assumptions of Theorem 4, we have

$$
\begin{aligned}
& \left|\chi_{\sigma}(x ; p)\left({ }_{\alpha}^{k} \mathbf{Z}_{\sigma} f g\right)(x ; p)-\left({ }_{\alpha}^{k} \mathbf{Z}_{\sigma} f\right)(x ; p)\left({ }_{\alpha}^{k} Z_{\sigma} g\right)(x ; p)\right| \\
& \leq\left|G_{\sigma, \sigma}\left(f, \zeta_{1}, \zeta_{2}\right)(x ; p) G_{\sigma, \sigma}\left(g, \eta_{1}, \eta_{2}\right)(x ; p)\right|^{\frac{1}{2}},
\end{aligned}
$$

where

$$
\begin{aligned}
G_{\sigma, \sigma}(m, n, o)(x ; p)= & \frac{\chi_{\sigma}(x ; p)\left[\left({ }_{\alpha}^{k} \mathbf{Z}_{\sigma}(n+o) m\right)(x ; p)\right]^{2}}{4\left({ }_{\alpha}^{k} \mathbf{Z}_{\sigma} n o\right)(x ; p)} \\
& -\left({ }_{\alpha}^{k} \mathbf{Z}_{\sigma} m\right)(x ; p)\left({ }_{\alpha}^{k} \mathbf{Z}_{\sigma} m\right)(x ; p) .
\end{aligned}
$$

Proof. By taking $\sigma=\varsigma$ in (30), we get the inequality (38). 
Corollary 4. If $\zeta_{1}=u, \zeta_{2}=U, \eta_{1}=v$ and $\eta_{2}=V$, then we have

$$
\begin{gathered}
\left|\chi_{\sigma}(x ; p)\left({ }_{\alpha}^{k} Z_{\sigma} f g\right)(x ; p)-\left({ }_{\alpha}^{k} Z_{\sigma} f\right)(x ; p)\left({ }_{\alpha}^{k} Z_{\sigma} g\right)(x ; p)\right| \\
\leq \frac{(U-u)(V-v)}{4 \sqrt{u U v V}}\left({ }_{\alpha}^{k} Z_{\sigma} f\right)(x ; p)\left({ }_{\alpha}^{k} Z_{\sigma} g\right)(x ; p) .
\end{gathered}
$$

Remark 7. In Theorem 4 and Theorem 5, for $\alpha(x)=x$ and $k=1$, we get [14] (Theorem 4, Corollary 4), for $\alpha(x)=x, k=1, \vartheta=p=0$ (and $a=0$ ), we get [10] (Theorem 3.6, Theorem 3.7).

\section{Conclusions}

We have proved some new Pólya-Szegö and Chebyshev type inequalities for generalized $k$-fractional integral operators involving Mittag-Leffler function in their kernels. The outcomes of this paper also provide a lot of Pólya-Szegö and Chebyshev type inequalities for several well-known fractional integral operators via parameter substitutions. The classical inequalities and results can be generalized by using the new $k$-fractional integral operators.

Author Contributions: Conceptualization, Z.Z.; G.F.; S.M.; K.N. and T.Y.; methodology, Z.Z.; G.F.; S.M.; K.N. and T.Y.; validation, G.F.; K.N. and T.Y.; writing-original draft preparation, G.F.; S.M. and K.N.; writing-review and editing, Z.Z.; G.F.; S.M.; K.N. and T.Y. All authors have read and agreed to the published version of the manuscript.

Funding: This research received funding support from the National Science, Research, and Innovation Fund (NSRF), Thailand.

Institutional Review Board Statement: Not applicable.

Informed Consent Statement: Not applicable.

Data Availability Statement: Not applicable.

Acknowledgments: We would like to thank the referees for their valuable comments and helpful advice on our manuscript.

Conflicts of Interest: The authors declare no conflict of interest.

\section{References}

1. Chebyshev, P.L. Sur les expressions approximatives des integrales definies par les autres prises entre les mêmes limites. Proc. Math. Soc. Charkov. 1882, 2, 93-98.

2. Hadamard, J. Etude sur les proprietes des fonctions entieres e.t en particulier dune fonction consideree par Riemann. J. Math. Pure Appl. 1893, 58, 171-215.

3. Jensen, J.L.W.V. Sur les fonctions convexes et les inégalités entre les valeurs moyennes. Acta Math. 1906, 30, 175-193. [CrossRef]

4. Pólya, G.; Szegö, G. Aufgaben und Lehrsatze aus der Analysis; Springer: Berlin, Germany, 1925.

5. Petrović, M. Sur une fontionnelle. Publ. Math. Univ. Belgrade 1932, 1, 146-149.

6. Grüss, G. Über das maximum des absolten Betrages von $\frac{1}{b-a} \int_{a}^{b} f(x) g(x) d x-\frac{1}{b-a} \int_{a}^{b} f(x) d x \int_{a}^{b} f(x) d x$. Math. Z. 1935, 39, 215-226. [CrossRef]

7. Ostrowski, A. Uber die Absolutabweichung einer differentiierbaren funktion von ihrem integralmittelwert. Comment. Math. Helv. 1937, 10, 226-227. [CrossRef]

8. Belarbi, S.; Dahmani, Z. On some new fractional integral inequalities. J. Inequal. Appl. Math. 2009, 10, 1-12.

9. Farid, G. Some new Ostrowski type inequalities via fractional integrals. Int. J. Anal. Appl. 2017, 14, 64-68.

10. Ntouyas, S.K.; Agarwal, P.; Tariboon, J. On Pólya-Szegö and Chebyshev types inequalities involving the Riemann-Liouville fractional integral operators. J. Math. Inequal. 2016, 10, 491-504. [CrossRef]

11. Sarikaya, M.Z.; Yildirim, H. On Hermite-Hadamard type inequalities for Riemann-Liouville fractional integrals. Miskolc Math. Notes 2016, 17, 1049-1059. [CrossRef]

12. Sarikaya, M.Z.; Set, E.; Yaldiz, H.; Basak, N. Hermite-Hadamard inequalities for fractional integrals and related fractional inequalities. J. Math. Comput. Model. 2013, 57, 2403-2407. [CrossRef]

13. Tariboon, J.; Ntouyas, S.K.; Sudsutad, W. Some new Riemann-Liouville fractional integral inequalities. Int. J. Math. Math. Sci. 2014, 2014, 6. [CrossRef]

14. Andrić, M.; Farid, G.; Mehmood, S.; Pečarić, J., Pólya-Szegö and Chebyshev types inequalities via an extended generalized Mittag-Leffler function. Math. Ineq. Appl. 2019, 22, 1365-1377. [CrossRef] 
15. Butt, S.I.; Akdemir, A.O.; Bhatti, M.Y.; Nadeem, M. New refinements of Chebyshev-Pólya-Szegö-type inequalities via generalized fractional integral operators. J. Inequal. Appl. 2020, 2020, 157. [CrossRef]

16. Dahmani, Z.; Mechouar, O.; Brahami, S. Certain inequalities related to the Chebyshev's functional involving a type RiemannLiouville operator. Bull. Math. Anal. Appl. 2011, 3, 38-44.

17. Deniz, E.; Akdemir, A.O.; Yüksel, E. New extensions of Chebyshev-Pólya-Szegö type inequalities via conformable integrals. AMIS Math. 2020, 5, 956-965. [CrossRef]

18. Farid, G.; Rehman, A.U.; Mishra, V.N.; Mehmood, S. Fractional integral inequalities of Grüss type via generalized Mittag-Leffler function. Int. J. Anal. Appl. 2019, 17, 548-558.

19. Rashid, S.; Jarad, F.; Kalsoom, H.; Chu, Y.M. Pólya-Szegö and Chebyshev types inequalities via generalized $k$-fractional integrals. Adv. Differ. Equ. 2020, 2020, 125. [CrossRef]

20. Wang, G.; Agarwal, P.; Chand, M. Certain Grüss type inequalities involving the generalized fractional integral operator. J. Inequal. Appl. 2014, 2014, 1-8. [CrossRef]

21. Dragomir, S.S.; Diamond, N.T. Integral inequalities of Grüss type via Pólya-Szegö and Shisha-Mond results. East Asian Math. J. 2003, 19, 27-39.

22. Agarwal, P. Some inequalities involving Hadamard-type k-fractional integral operators. Math. Methods Appl. Sci. 2017, 40, 3882-3891. [CrossRef]

23. Agarwal, P.; Jleli, M.; Tomar, M. Certain Hermite-Hadamard type inequalities via generalized $k$-fractional integrals. J. Inequal. Appl. 2017, 2017, 1-10. [CrossRef]

24. Akkurt, A.; Yildirim, M.E.; Yildirim, H. On some integral inequalities for $(k, h)$-Riemann-Liouville fractional integral. New Trends Math. Sci. 2016, 4, 138-146. [CrossRef]

25. Andrić, M.; Farid, G.; Pečarić, J. Analytical Inequalities for Fractional Calculus Operators and the Mittag-Leffler Function; Element: Zagreb, Croatia, 2021.

26. Chen, H.; Katugampola, U.N. Hermite-Hadamard and Hermite-Hadamard-Fejér type inequalities for generalized fractional integrals. J. Math. Anal. Appl. 2017, 446, 1274-1291. [CrossRef]

27. Habib, S.; Mubeen, S.; Naeem, M.N. Chebyshev type integral inequalities for generalized $k$-fractional conformable integrals. $J$. Inequal. Spec. Func. 2018, 9, 53-65.

28. Kwun, Y.C.; Farid, G.; Latif, N.; Nazeer, W.; Kang, S.M. Generalized Riemann-Liouville $k$-fractional integrals associated with Ostrowski type inequalities and error bounds of Hadamard inequalities. IEEE Access 2018, 6, 64946-64953. [CrossRef]

29. Mehmood, S.; Farid, G.; Khan, K.A.; Yussouf, M. New fractional Hadamard and Fejér-Hadamard inequalities associated with exponentially $(h, m)$-convex function. Eng. Appl. Sci. Lett. 2020, 3, 9-18. [CrossRef]

30. Khan, T.U.; Khan, M.A. Generalized conformable fractional operators. J. Comput. Appl. Math. 2019, 346, 378-389. [CrossRef]

31. Che, Y.; Keir, M.Y.A. Study on the training model of football movement trajectory drop point based on fractional differential equation. Appl. Math. Nonlinear Sci. 2021, 1-6. [CrossRef]

32. Farid, G. A unified integral operator and further its consequences. Open J. Math. Anal. 2020, 4, 1-7. [CrossRef]

33. Jarad, F.; Ugurlu, E.; Abdeljawad, T.; Baleanu, D. On a new class of fractional operators. Adv. Differ. Equ. 2017, 2017, 247. [CrossRef]

34. Man, S.; Yang, R.; Educational reform informatisation based on fractional differential equation. Appl. Math. Nonlinear Sci. 2021, 1-10. [CrossRef]

35. Zhao, N.; Yao, F.; Khadidos, A.O.; Muwafak, B.M. The impact of financial repression on manufacturing upgrade based on fractiona Fourier transform and probability. Appl. Math. Nonlinear Sci. 2021, 1-11. [CrossRef]

36. Andrić, M.; Farid, G.; Pečarić, J. A further extension of Mittag-Leffler function. Fract. Calc. Appl. Anal. 2018, 21, 1377-1395. [CrossRef]

37. Salim, T.O.; Faraj, A.W. A generalization of Mittag-Leffler function and integral operator associated with integral calculus. J. Frac. Calc. Appl. 2012, 3, 1-13.

38. Rahman, G.; Baleanu, D.; Qurashi, M.A.; Purohit, S.D.; Mubeen, S.; Arshad, M. The extended Mittag-Leffler function via fractional calculus. J. Nonlinear Sci. Appl. 2017, 10, 4244-4253. [CrossRef]

39. Srivastava, H.M.; Tomovski, Z. Fractional calculus with an integral operator containing generalized Mittag-Leffler function in the kernal. Appl. Math. Comput. 2009, 211, 198-210.

40. Prabhakar, T.R. A singular integral equation with a generalized Mittag-Leffler function in the kernel. Yokohama Math. J. 1971, 19, 7-15.

41. Kilbas, A.A.; Srivastava, H.M. Trujillo, J.J. Theory and Applications of Fractional Differential Equations; North-Holland Mathematics Studies; Elsevier: New York, NY, USA; London, UK, 2006.

42. Sarikaya, M.Z.; Dahmani, M.; Kiris, M.E.; Ahmad, F. $(k, s)$-Riemann-Liouville fractional integral and applications. Hacet. J. Math Stat. 2016, 45, 77-89. [CrossRef]

43. Mubeen, S.; Habibullah, G.M. k-fractional integrals and applications. Int. J. Contemp. Math. Sci. 2012, 7, 89-94. 